\title{
SHORT REPORT
}

\section{Dopaminergic deficit in amyotrophic lateral sclerosis assessed with [I-123] IPT single photon emission computed tomography}

\author{
G D Borasio, R Linke, J Schwarz, V Schlamp, A Abel, P D Mozley, K Tatsch
}

A Abel

Department of

Nuclear Medicine,

Klinikum

Grosshadern,

Ludwig-Maximilians-

Universität, D-81366

München, Germany

$\mathrm{R}$ Linke

K Tatsch

Department of

Radiology, University

of Pennsylvania,

Philadelphia, PA, USA

P D Mozley

Correspondence to:

Dr G D Borasio,

Department of Neurology,

University of Munich,

Klinikum Grosshadern,

D-81366 München,

Germany. Telephone 0049

897095 3671; fax 004989

7095 3677; email

Borasio@lrz.uni-muenchen.de

Received 2 July 1997 and in revised form 20 November 1997

Accepted 25 February 1998

Keywords: dopamine transporter; basal ganglia; motor neuron disease; single photon emission tomography

Similarities between amyotrophic lateral sclerosis (ALS) and Parkinson's disease suggest possible common traits in their pathogenesis. The incidence of both disorders increases with age, their onset is gradual, and their course relentlessly progressive. The underlying pathological process is thought in both cases to begin long before the appearance of clinical symptoms, and there are indications of a shared genetic susceptibility. ${ }^{1}$ At postmortem examination, the brains of patients with ALS and patients with Parkinson's disease share several features, including accumulation of neurofilamentous material in damaged neurons. ${ }^{2}$ A prominent example of association between these disorders is the ALS-parkinsonismdementia complex, once highly prevalent in the western Pacific. Neuropathological ${ }^{3}$ and $\mathrm{PET}^{45}$ studies have provided evidence for an involvement of the dopaminergic system in ALS. The purpose of this study was to investigate whether patients with ALS show presynaptic nigrostriatal dopaminergic deficits de-

\begin{abstract}
Department of
Neurology

G D Borasio

V Schlamp

Abstract

Dopamine transporter imaging was performed in 18 patients with sporadic amyotrophic lateral sclerosis (ALS) and 11 age matched controls with [I-123] IPT (N-(3-

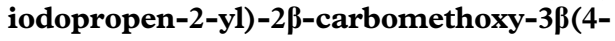
chlorophenyl)-tropane), a new cocaine analogue that selectively binds to the dopamine transporter located on dopaminergic nerve terminals. Image analysis showed that striatal IPT binding was moderately but significantly reduced in the ALS group compared with controls $(p<0.01)$. The reduction of IPT binding was similar for patients with bulbar onset compared with those with limb onset. There was no correlation between values for uptake of striatal IPT and the age of the patients or the duration of the disease. These data indicate that nigrostriatal dopaminergic neurons are subclinically affected in a subset of patients with sporadic ALS.

(F Neurol Neurosurg Psychiatry 1998;65:263-265)
\end{abstract}

tectable by single photon emission computed tomography (SPECT).

\section{Materials and methods}

We investigated 18 patients (age $42-79$, mean 62.5 (SD 10.5) years.; disease duration 4-107 months, mean 38.7 months) with sporadic ALS, from the motor neuron disease clinic of the Department of Neurology, University of Munich, and 11 age matched controls (age 41-81, mean 61.2 (SD 12.1) years). All patients fulfilled the El Escorial criteria of the World Federation of Neurology for probable or definite ALS. None of the patients showed signs of dementia, parkinsonism, or ophthalmoplegia by clinical examination. The protocol for the SPECT study was approved by the local ethics committee and signed informed consent was obtained from the patients.

$\mathrm{N}$-(3-iodopropen-2-yl)-2 $\beta$-carbomethoxy$3 \beta$-(4-chlorophenyl)tropane (IPT) was labelled with $\left[{ }^{123} \mathrm{I}\right]$ iodine as described previously. ${ }^{6}$ The sodium $\left[{ }^{123} \mathrm{I}\right]$ iodide was obtained commercially(Cygne BV, Eindhoven, The Netherlands). The radionuclidic purity of the ${ }^{123} \mathrm{I}$ was $>99.9 \%$ and the specific activity was calculated to be $2 \times 10^{17} \mathrm{~Bq} / \mathrm{mol}$. Data acquisition and processing has been described in detail. ${ }^{7}$ Briefly, the patients were injected with 150 to $180 \mathrm{MBq}$ of radioactively labelled IPT in an antebrachial vein. Brain SPECT images were acquired from 90 to 120 minutes after injection. Previous studies have shown that this period (corresponding to the washout phase after peak activity has been reached) may be suitable to clearly discriminate between patients with striatal disease and controls. ${ }^{7}$ For acquisition a triple headed gamma camera (Picker Prism 3000) equipped with high resolution fan beam collimators was used. The acquisition parameters comprised a rotational radius of $\leqslant 13.2$ $\mathrm{cm}$, a $20 \%$ energy window centred on $159 \mathrm{keV}$, 120 projection angles over $360^{\circ}$, an acquisition time of 45 seconds per projection, and a $128 \times 128$ matrix with a pixel width of $2.11 \mathrm{~mm}$ in the projection domain. The projection images were reconstructed by filtered back projection and further processed with a count rate dependent restorative (Wiener) filter. For uniform attenuation correction Chang's first order 
method was used. Images were resliced parallel to the AC-PC line.

To assess specific tracer uptake in the striatum we used the region of interest (ROI) technique. ROIs of the striatum, caudate nucleus, and putamen were defined by templates established from the data set of the control group. Non-specific activity was estimated by drawing a large ROI around the entire supratentorial brain on the slices containing the basal ganglia, which excluded the basalganglia and the thalamus. Mean specific activity in

Table 1 Specific IPT binding rates

\begin{tabular}{lrllll}
\hline & \multicolumn{1}{c}{$n$} & $((S-B G) / B G)$ & $((C-B G) / B G)$ & $((P-B G) / B G)$ & $P / C$ \\
\hline Controls & 8 & $7.1(1.1)$ & $8.3(1.3)$ & $6.4(1.1)$ & 0.78 \\
ALS patients & 18 & $5.6(1.1)^{\star \star}$ & $6.8(1.4)^{\star}$ & $4.8(1.1)^{\star \star}$ & 0.71 \\
$\quad$ Limb onset & 10 & $5.8(1.2)$ & $6.9(1.5)$ & $4.9(1.3)$ & 0.71 \\
Bulbar onset & 8 & $5.5(1.0)$ & $6.6(1.2)$ & $4.6(0.9)$ & 0.71 \\
\hline
\end{tabular}

${ }^{\star} \mathrm{p}<0.01 ;{ }^{\star \star} \mathrm{p}<0.005 v$ controls. Data are given as mean (SD)

$\mathrm{ALS}=\mathrm{ALS} ; \mathrm{S}=$ striatum $; \mathrm{C}=$ caudate $\mathrm{P}=$ putamen; $\mathrm{BG}=$ background

basal ganglia regions was calculated by subtracting the mean counts per pixel in the background (BG) from the mean counts per pixel in the basal ganglia regions and dividing the results by the mean counts per pixel in the background ((ROI-BG)/BG). The table shows the IPT uptake ratios measured in patients and controls. Statistical significance was assessed with Student's $t$ test (two tailed).

\section{Results}

Image analysis showed that the specific striatal uptake of IPT was diminished $>1$ SD below the control mean in 12 out of 18 patients with ALS, and $>2$ SD below control mean in four out of 18 patients with ALS. The reduction in IPT uptake was similar for both putamen and caudate nucleus. Striatal IPT binding was moderately but significantly reduced in the ALS group compared with controls $(\mathrm{p}<0.005)$. Figure 1 shows the individual data for patients and controls. The reduction of IPT binding was similar for patients with bulbar onset com- pared with those with limb onset (table). There was no correlation between striatal IPT uptake values and patient age, sex, or duration of the disease (fig 2). The linear correlation values were as follows: disease duration versus striatal uptake, $r=0.23, \mathrm{p}=0.36$; age versus striatal uptake (patients) $: r=0.40, \mathrm{p}=0.10$. The control group showed an age dependency of striatal uptake values $(r=0.69 ; \mathrm{p}=0.02)$, as previously described. ${ }^{6}$ This correlation was weaker, but still significant, in the total group (patients and controls: $r=0.46 ; \mathrm{p}=0.01$ ).

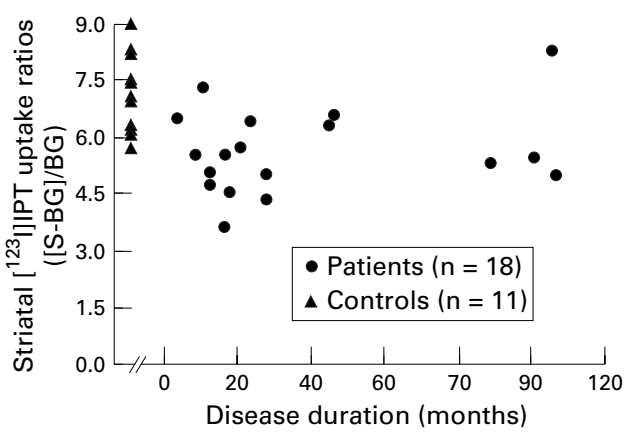

Figure 1 Striatal IPT uptake ratios of patients and controls. No correlation can be seen between specific striatal IPT binding and disease duration.

\section{Discussion}

In ALS, structures distinct from the upper and lower motor neurons, such as the prefrontal cortex, the spinocerebellar and dorsal tracts, show subclinical neuropathological involvement in some but not all patients. Neuropathological damage to the substantia nigra of patients with ALS has been previously documented. ${ }^{3}$ However, many of these patients showed atypical clinical features such as parkinsonism, dementia, or ophthalmoplegia. Although the concomitant occurrence of ALS and parkinsonian features in the same patient is reportedly more frequent than expected by chance, ${ }^{8}$ this does not seem to be true for ALS and idiopathic Lewy body Parkinson's disease. ${ }^{9}$
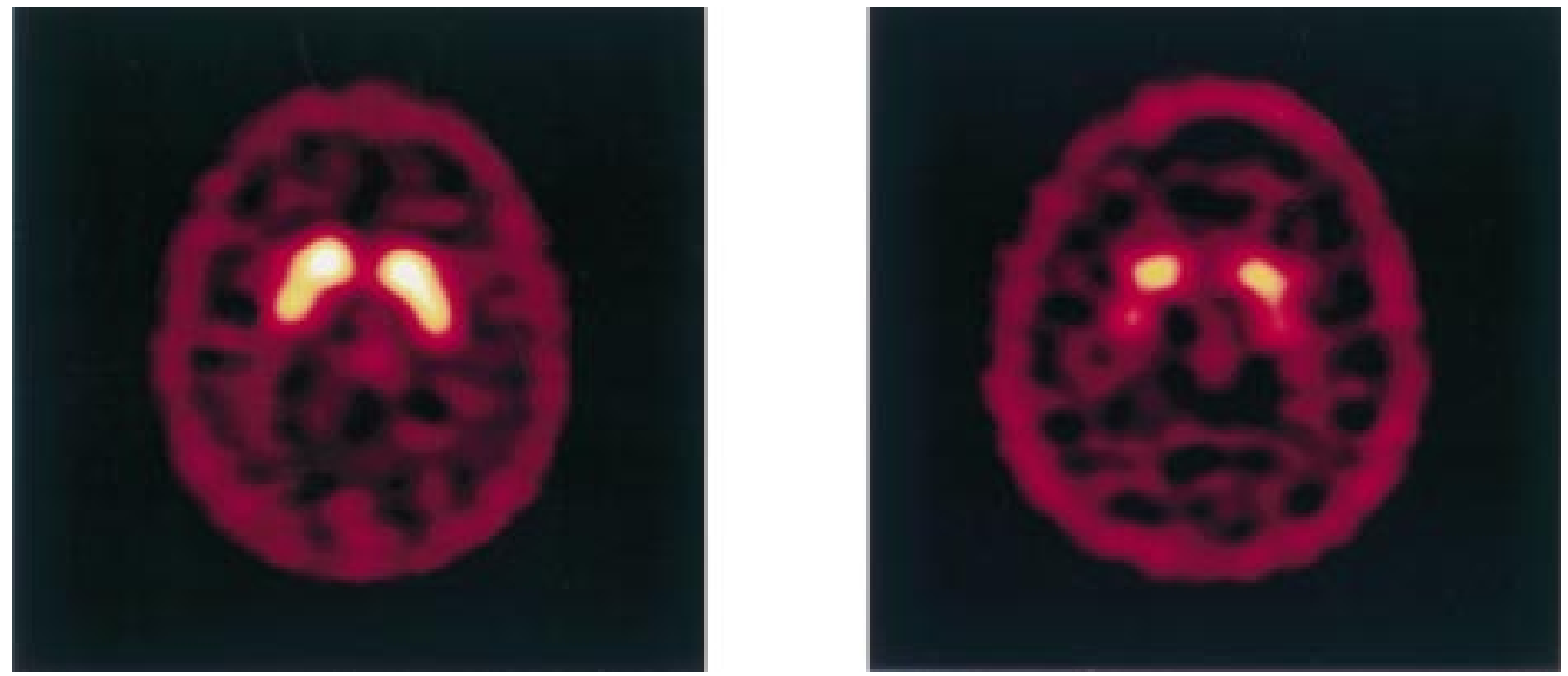

Figure 2 IPT-SPECT of two patients with ALS. The patient on the right exhibits a marked decrease in striatal IPT binding ([S-BG]/BG $=4.4 ;$ disease duration 28 months), whereas the patient on the left shows IPT binding in the upper normal range $([S-B G] / B G=8.3)$, despite a disease duration of 106 months. 
Presynaptic dopaminergic nerve terminals have usually been investigated with fluorodopa-PET, which primarily reflects levodopa metabolism. Dopamine transporter binding might provide a more accurate tool for estimating the number of dopaminergic neurons. This hypothesis is corroborated by recent data from a primate model showing that in vivo quantification of dopamine transporter binding strongly correlates with the number of striatal dopaminergic nerve terminals as assessed by postmortem autoradiography. ${ }^{10}$ However, the possibility that down regulation or altered post-translational processing of the dopamine transporter might contribute to the decreased striatal IPT binding cannot be ruled out.

The imaging method and characteristics of IPT have been described in detail previously. ${ }^{6}{ }^{711} 12$ The uptake ratio used in our study may serve as a measure of specific IPT binding to striatal structures. With our study design no attempt was made to prove that outcome measures are linearly related to the density of dopamine transporters. As measurements were performed during the washout phase of striatal activity, outcome measures theoretically could have non-linearly overestimated differences between patients and controls.

In a PET study on 16 patients with ALS, Takahashi et $a l^{4}$ found a significant negative correlation between 6-fluorodopa uptake and duration of disease. Four patients had an uptake rate $<2 \mathrm{SD}$ below the control mean, whereas the mean uptake was not significantly different from the control value. We did not find any correlation with disease duration, despite the inclusion of four patients with a prolonged clinical course. This is in agreement with recent data from patients with familial ALS. ${ }^{5}$ However, due to the few patients, we cannot completely exclude the possibility of a correlation between dopaminergic damage and disease duration in ALS. The overlap between patients and controls seen in both our study and the previous one is consistent with the subclinical nature of the involvement of the dopaminergic system in ALS. Interestingly, although transgenic SOD1 mice display midbrain dopaminergic degeneration, ${ }^{13}$ patients with familial ALS carrying SOD1 mutations were less likely to show dopaminergic damage as compared to non-SOD1 patients with familial ALS.

Taken together, our data suggest a heterogeneity in the ALS patient population, with a significant subset of patients whose disease subtype is more likely to affect nigrostriatal dopaminergic cells. This hypothesis is consistent with the well known genetic heterogeneity in familial ALS, implying that numerous types of chronic damage to motor neurons can ultimately lead to the common final pathway of the disease. Follow up measurements are currently in progress to corroborate and extend the findings of this pilot study.

1 Majoor-Krakauer D, Ottman R, Johnson WG, et al. Familial aggregation of amyotrophic lateral sclerosis, dementia, and Parkinson's disease: evidence of shared genetic susceptibilParkinson's disease: evidence of

ity. Neurology 1994;44:1872-7.
2 Jellinger K. Cytoskeletal pathology of parkinsonism and aging brain. In: Calne DB, Comi G, Grippa D, et al, eds. Parkinsonism and aging. New York: Raven Press, 1989:3556

3 Kato S, Oda M, Tanabe H. Diminution of dopaminergic neurons in the substantia nigra of sporadic amyotrophic lateral sclerosis. Neuropathol Appl Neurobiol 1993;19:300-4.

4 Takahashi H, Snow BJ, Bhatt MH, et al. Evidence for a dopaminergic deficit in sporadic amyotrophic lateral sclerosis on positron emission scanning. Lancet 1993;342: 1016-18.

5 Przedborski S, Dhawan V, Donaldson DM, et al. Nigrostriatal dopaminergic function in familial amyotrophic lateral sclerosis patients with and without copper/zinc superoxide dismutase mutations. Neurology 1996;47:1546-51.

6 Mozley PD, Kim HJ, Gur RC, et al. Iodine-123-IPT SPECT imaging of the CNS dopamine transporters: nonlinear effects of normal aging on striatal uptake values. $\mathfrak{F}$ Nucl Med 1996;37:1965-70.

7 Tatsch K, Schwarz J, Mozley PD, et al. Relationship between clinical features of Parkinson's disease and presynaptic dopamine transporter binding assessed with $\left[{ }^{123} \mathrm{I}\right] \mathrm{IPT}$ and single-photon emission tomography. Eur F Nucl Med 1997; 24:415-21.

8 Eisen A, Calne DB. Amyotrophic lateral sclerosis, Parkinson's disease, and Alzheimer's disease: phylogenetic disorders of the human neocortex sharing many characteristics. Can f Neurol Sci 1992;19:117-20.

9 Williams TL, Shaw PJ, Lowe J, et al. Parkinsonism in motor neuron disease: case report and literature review. Acta Neuropathol (Berl) 1995;89:275-83.

10 Hoffmann JM, Shi B, Goodman MM, et al. Correlation of in-vivo and in-vitro dopamine transporter function. Neurology 1997;48(suppl):A62-3.

11 Mozley PD, Stubbs JB, Kim HJ, et al. Dosimetry of an iodine-123-labeled tropane to image dopamine transportodine-123-labeled tropane to ima
ers. F Nucl Med 1996;37:151-9.

12 Kung MP, Essman WD, Frederick D, et al. IPT: a novel iodinated ligand for the CNS dopamine transporter. Synapse 1995;20:316-24.

13 Kostic V, Gurney ME, Deng HX, et al. Midbrain dopaminergic neuronal degeneration in a transgenic mouse model of familial amyotrophic lateral sclerosis. Ann Neurol 1997;41:497-504. 